\title{
Crystalline-silicon-based infra-red LEDs and routes to laser diodes
}

\author{
M. A. Lourenço, K. P. Homewood* \\ Advanced Technology Institute, Faculty of Engineering and Physical Sciences, University of Surrey, \\ Guildford, Surrey, GU2 7XH, UK
}

\begin{abstract}
We review progress in silicon LEDs using dislocation engineering to achieve high temperature operation, a process that is fully CMOS (Complementary Metal Oxide Semiconductor) compatible. We concentrate on devices operating in the near infra red where high value applications are. The need for silicon emitters, lasers and optical amplifiers is discussed followed by an outline of previous approaches and possible future routes explored. Results on gain in silicon are reported and routes to electrically pumped injection lasers and optical amplifiers considered. Extension of 1.1 and $1.5 \mu \mathrm{m}$ devices to other wavelengths is discussed.

Keywords: Dislocation engineering; Light-emitting diodes; Rare earth compounds; Silicon
\end{abstract}

*Corresponding author. Tel.: +44-(0)1483689285, fax.: +44-(0)1483686091

E-mail address: k.homewood@ surrey.ac.uk 


\section{Introduction}

1.1 The need for light sources, LEDs, lasers and optical amplifiers in silicon

Silicon is the primary semiconductor material and represents around $90 \%$ of the semiconductor

chip market. Ultra-large-scale-integration (ULSI) technology of silicon is the basis of microelectronics and drives the exponential growth (Moore's Law [1]) in computer speed and power that underpins the whole information technology sector. Despite its predominance, silicon lacks a key semiconductor property. It has an indirect band-gap and is fundamentally unable to emit light. Indeed, the light emitting process in silicon is described as forbidden! Nevertheless, it is increasingly clear that we need light emitting and light amplifying devices on silicon.

Direct gap semiconductors, in particular those based on III-V materials such as GaAs, dominate the LED and Laser Diode sectors and currently provide the light emission technology that supports optical communication systems, optical disc data storage and retrieval for computer drives, audio and video systems. It would seem at first sight that when silicon "needs light" the obvious approach would be to hybridise III-V light emitters with conventional silicon technology (the "stick a laser diode onto silicon" approach). Indeed, this method has and can be used in high added value systems, for example in optical communication platforms. However, applying hybridised systems to silicon integrated circuits such as microprocessor chips of high volume, ultra small feature sizes and high packing density is technically difficult and involves a considerable increase in production 
complexity and cost. The ideal solution would be an emitter based in silicon itself and this is the reason for the intensive interest and research activity to achieve this end over the last fifteen years. A particular driver has been the recognition of a fundamental problem in microelectronics - the so called interconnect problem.

The exponential growth in computer speed and power in microprocessor chips for the past three decades has resulted from a simple scaling down of the gate length of the active transistors at each technology node, typically each 18-24 months. The transistor physics will continue to scale for several more generations. However, the electrons must move between these transistors in metal interconnections, and this takes a small but finite time, which does not scale at all. Consequently, rather than doing anything functionally useful, as the transistor is made smaller, more and more time is spent by electrons moving around the chip. This provides an ultimate and imminent limit to computer speed. The recognized solution to this problem is optical data transfer on chip for some functions such as the clock. The optimum solution to this is an optical emitter in silicon itself. A light emission technology in silicon would also offer other applications such as inter-chip optical links, all-silicon optical communications, optical micro-electro-mechanical systems (OMEMS) in silicon, optical computing, sensors, etc. All of these applications would benefit from the large cost savings associated with the cheaper silicon substrate and processing.

Hybrid lasers are used and can provide a light source for optical signaling on silicon although, 
as discussed above, this is not the optimum solution. It has also been suggested that a laser integrated on chip is not necessary and could be external to the chip and just provide a source of photons for a photonic circuit or chip, analogous to how a power supply supplies electrons to an electronic circuit or chip. This is possibly true but, of course, the light once on chip is quickly attenuated as it is distributed, modulated, switched, etc. Regenerative amplification is not a viable route as it is too slow. Optical amplifiers integrated on chip are therefore, in some ways, the more important and indeed essential device for the implementation of any practical silicon photonics platform. Optical amplifiers are single pass devices and by their nature need to be inserted with low, ideally zero, coupling loss and no back or forward reflections in to the photonic circuit. A satisfactory hybrid solution to this problem is highly problematic and probably impossible. Again the ideal solution would be a silicon optical amplifier fully integrated in to the silicon waveguide.

The past few years has seen tremendous advances in the development and performance of all the other silicon photonic devices [2-12] required for a silicon photonics platform. However, the last remaining hurdle to the implementation of full silicon photonic systems and optical data transfer on and between integrated circuits remains the need to develop electrically pumped optical amplifiers and lasers in silicon using a CMOS compatible technology. Consequently, there has been a massive effort worldwide to search for efficient light emission from silicon, starting with LEDs for lower cost lower performance systems such as sensors but with the ultimate aim being a silicon laser for high 
value high performance applications. The importance to the microelectronics and telecommunications industries of the realization of such devices and full silicon photonic platforms is difficult to overestimate.

A key constraint on silicon light emission technology is that, because of the large investment in the microelectronics industry, any new approach needs to be closely compatible with silicon ULSI technology. The challenge then is to produce an efficient light emitter which uses just the standard ULSI tools such as ion implantation and materials such as silicon and boron. Dislocation engineering (DE), a method developed to fabricate efficient silicon light emitting diodes operating at room temperature [3], uses ion implantation at standard energies and doses, and simple dopants such as boron and so, crucially, is completely compatible with conventional silicon technology.

1.2 State of the art and future challenges for silicon LEDs and lasers

The obvious benefits of an efficient silicon-based LED have resulted in very considerable research activity particularly over the last decade. Much interest was created by the discovery of light emission from porous silicon in 1990 [13] which generated a considerable amount of subsequent activity. Porous silicon based devices emit primarily in the visible and so we will not discuss these further in this paper. For interested readers this has been summarised in many reviews on the subject [14]. 
Other approaches attempted include the use of silicon-germanium superlattice structures with a periodicity designed to fold the indirect gap in to centre of the Brillouin zone to create a quasi-direct semiconductor system [15]. However, insufficient enhancement of the matrix element for the quasi-direct transition was achieved to make this a viable approach for efficient LEDs. A large and ongoing activity is based on incorporating nano-silicon in glassy matrices. The main issues here are getting sufficient current injection in what is an insulating host. These devices are operated under high reverse bias fields which restrict the power efficiencies and also lead to device degradation. Again there are many reviews on the developments in this area [16]. The incorporation of rare earths in both oxide matrices, with and without silicon nanocrystals [17], and also in bulk silicon [18-21] have been also extensively investigated. These devices rely on exciting the internal transitions of the rare earth which can be chosen to give emission in either the visible or the infra-red. In the infra-red the rare earth erbium has been of particular interest and we will come back to this later in this paper.

A group at the University of Surrey made a major breakthrough producing the first light emitting diode fabricated in bulk silicon by incorporating the direct gap semiconductor material iron disilicide [22]. Although a world first this device only operated efficiently at low temperatures. Many groups are still involved in research on and improving the performance of iron disilicide based light emitting devices [23]. The same Surrey group subsequently developed a new approach dislocation engineering - to eliminate the problem of thermal quenching in silicon LEDs [3]. The DE 
method was used to fabricate silicon LEDs operating efficiently at room temperature and, crucially,

using only conventional CMOS technology.

Optical gain in crystalline silicon has been reported utilizing the Raman effect [6-8] and phase matched four wave mixing [10]. Although significant, these effects are purely optical and, for fundamental reasons, neither are capable of being electrically pumped. There is one report of optically pumped lasing in crystalline silicon [9]. The emission occurs at a wavelength of $1.27 \mu \mathrm{m}$, was attributed to the so called A-centre, subsequently identified as the G-centre, and was introduced using surface texturing. Again this is a significant result but it is unclear whether this centre could be incorporated controllably using CMOS compatible processes.

The development of an LED technology into a silicon injection laser and electrically driven optical amplifiers is an essential next step for high technology high value applications. For any semiconductor to lase net gain (gain $>$ losses) is required in an optical cavity. Gain can only be achieved by the mechanism of population inversion. The primary challenge in silicon is to identify a suitable gain/population inversion mechanism (waveguide cavities have already been demonstrated in silicon and is a relatively simple and mature technology). The dislocation engineering approach demonstrates that we can eliminate thermal quenching leading to room temperature light emission from an LED under forward bias. All conventional semiconductor laser diodes operate in this mode. It should be noted that the dislocation engineered method does not in itself provide an intrinsic gain 
mechanism. What it can provide is carrier confinement and reduction of the nonradiative losses in the device and so give the potential for room and higher temperature operation. To get gain and ultimately lasing or amplification, in addition, population inversion in the active device region must be achieved.

One can identify at least four possible approaches to achieve population inversion and gain in bulk silicon:

(i) Conventional: Conventional semiconductor amplifiers and laser diodes rely on degenerate (very high) doping of the $\mathrm{p}$ and $\mathrm{n}$ regions of the device and overlapping these populations in the $\mathrm{p}-\mathrm{n}$ junction to provide the population inversion. This approach could work in principle in silicon if sufficient gain can be achieved to overcome the loss mechanisms associated with the high doping, such as free carrier absorption and auger recombination. However, the required high gain is probably only achievable in direct gap semiconductors where radiative transition rates are high, so making this the most unlikely route in silicon.

(ii) Phonon assisted: Silicon is an indirect gap material, the absorption process requires the addition of a phonon and the emission process the generation of a phonon; this provides a four level system that gives a route to intrinsic population inversion and gain [24]. It should be possible to pump this transition using lightly doped device structures that would reduce the free carrier absorption and auger recombination. This question has only previously been addressed theoretically 
and results are contradictory [25].

(iii) Additional optical centre: The use of an additional optically active centre that can itself provide population inversion. For example a number of rare earths are able to sustain lasing and can provide wavelength emission at wavelengths in the extended optical communications band of 1.2 $1.8 \mu \mathrm{m}$. Again these centres should be able to be electrically pumped in more lightly doped device structures. Although such systems are often low gain they can also be incorporated in to low loss devices, in principle enabling net optical gain. For example the rare earth erbium is excited electrically in silicon via band gap recombination and the elimination of thermal de-excitation and room temperature electroluminescence (EL), under forward bias, has been reported in dislocation engineered samples [26]. Erbium in the $\mathrm{Er}^{3+}$ state possesses intrinsic, internal, gain and is the basis of the erbium fibre amplifier.

(iv) The G-centre: This is similar to (iii) and has been recently demonstrated to support lasing in silicon $[9,27]$. This device was optically pumped and operated only at low temperatures; the G-centre was introduced by a surface texturing technique. The combination of this centre - which should be able to be introduced using ion implantation - and the DE method may be able to provide a route to room temperature operation.

(v) Tensile strained germanium: This approach is not strictly a route to a true silicon laser but is nevertheless relevant because the use of germanium in state-of-the-art CMOS is becoming 
increasingly common. This system incorporates germanium grown off silicon so that tensile strain is incorporated in the germanium layer [28]. Under these conditions the upper direct gap in the germanium is lowered with respect to the indirect minima allowing the direct gap to be partially populated by carrier transfer from the indirect gap under sufficiently high carrier injection, enabling a direct transition to occur. Theoretical analysis of this system indicates the possibility that it might lead to lasing [29].

The remainder of this paper will consider on evaluating the potential of route (iii), the DE approach along with the incorporation of optically active centres that have the potential to give gain and offer a route to lasing and optical amplification in silicon. We will describe some of the recent progress in these areas.

\section{Results and Discussion}

Conventional semiconductor light emitting diodes suffer from thermal quenching; that is, the efficiency drops as the operating temperature increases. The thermal quenching is due to the presence of competing nonradiative recombination through defects - the radiative recombination route is in fact temperature independent to first order. This problem is particularly severe in indirect gap materials where the radiative transition is forbidden or, in practice, has a very long radiative recombination lifetime. In contrast, in the dislocation engineered light emitting diodes (DELEDs) the 
thermal quenching is largely reduced or completely eliminated. The paramount importance of DE in enabling light emission in silicon is shown in Fig. 1.

The dislocation engineering approach has been described in detail elsewhere [3,30-37] so will only be briefly outlined here. DE is based on the controlled incorporation of dislocation loops to form arrays of loops in critical regions of the device. The presence of the loops distorts the local surroundings which become strained.

The significance of the introduction of a strain field in the silicon lattice is that the band structure, and in general the band energies of all semiconductors, are strain dependent. Consequently if the strain within a semiconductor material or device can be controllably modified, then so can the band gap energies, enabling potential variations to be introduced in the conduction and valence bands to control carrier migration. For silicon, the pressure dependence of the minima in the conduction band has a negative pressure coefficient so outside the loop the band gap is increased. Consequently, a loop repels carriers from itself and can be used to "shepherd" the carriers around a device. This loop technology has been employed to form dislocation engineered silicon LEDs. Conventional dislocation engineered light emitting diodes operate with an emission wavelength at the short wavelength end of the extended optical communication band, $1.2 \mu \mathrm{m}$. A $1.5 \mu \mathrm{m}$ DELED that operates at room temperature [26] has been already demonstrated, based on the rare earth erbium, There is also considerable interest in and need for other wavelengths in the extended optical 
communications band from 1.2 to $1.8 \mu \mathrm{m}$ for optical fibre communications and, in particular, an emitter at $1.3 \mu \mathrm{m}$ is required in key applications such as Fibre to the Home (FTTH). One way to obtain these wavelengths is to use other optically active materials or centres in silicon - this approach has previously always failed in bulk silicon due to the thermal quenching. However, as in a DELED the active region of the device is decoupled from the regions containing nonradiative centres, optically active materials or centres introduced in to the active region between the depletion region edge and the loop array should not be affected by silicon related nonradiative recombination. The emission spectrum observed should then just be determined by the relative rates of the optical centre emission and the radiative silicon transition. Recently, bulk silicon light emitting diodes operating over the 1.2 to $1.35 \mu \mathrm{m}$ range [38] have been demonstrated. This was achieved by the implantation of the rare earth thulium, incorporated in the trivalent $\mathrm{Tm}^{3+}$ state, into silicon $\mathrm{p}-\mathrm{n}$ junctions. Light emitting diodes operating under forward bias have been obtained by co-doping of boron to reduce the thermal quenching. Seven sharp lines were observed, corresponding to known internal $\mathrm{Tm}^{3+}$ transitions in the manifold from the ${ }^{3} \mathrm{H}_{5}$ to the ${ }^{3} \mathrm{H}_{6}$ ground state. Fig. 2 shows electroluminescence results on this system. The LEDs were operated at a forward bias of $1 \mathrm{~V}$ and current of $1.25 \mathrm{~A} / \mathrm{cm}^{2}$.

The first ever measurements of optical gain at $1.5 \mu \mathrm{m}$ in crystalline silicon have recently been reported [39]. Gain was achieved by the incorporation of the rare earth erbium in silicon. A new method [39] needed to be developed to enable the gain measurement in short silicon waveguides. Fig. 
3 shows the photoluminescence from an Er-doped silicon waveguide with only the probe laser on and with both probe and pump lasers on. Also shown in the inset is the enhancement (gain) in the luminescence over the erbium spectral region (1500-1700 nm) under optical pumping. The gain values obtained were found to be significantly greater than previously supposed. A measured lower limit for the optical cross section for $\mathrm{Er}^{3+}$ of $5 \times 10^{-19} \mathrm{~cm}^{2}$ was found, thirty times higher than previously anticipated [40]. Crucially, it was also shown that the gain significantly exceeds any losses due to the additional free carriers needed to achieve it.

Fig. 4 shows the power density and temperature dependence of the gain. The power dependence of the gain could be fitted without the inclusion of terms for co-operative up-conversion and excited state absorption, showing that these loss mechanisms are negligible in the Er:Si system. In addition, from the temperature dependence data it can be seen that the introduction of the dislocation loops has significantly reduced the thermal quenching. The residual quenching in the sample is attributed to erbium ions in the tail of the implants above the loop array. Currently work is being carried out to increase the incorporation of erbium in silicon and have already managed to achieve optically active values of $10^{19} \mathrm{~cm}^{-3}$. At this Er concentration one would expect to obtain a net material gain greater than $20 \mathrm{~dB} \mathrm{~cm}^{-1}$. Given these higher values, this system would seem to offer a realistic route to the production of electrically pumped silicon optical amplifier and laser devices using standard silicon process technology. 


\section{Conclusions}

We have outlined the need for and routes to achieving infrared silicon based LEDs capable of being fully integrated in to all-silicon optical platforms and ULSI chips. This paper concentrated on a core technology - dislocation engineering - which is rather unique in being totally CMOS compatible. We also discussed how silicon laser and optical amplifier technology could be developed from the basic dislocation engineered LED device when centres with intrinsic gain such as rare earths are introduced. We reported recent results showing unusually high optical cross-section of Er in silicon, indicating that significant gain of $\sim 20 \mathrm{~dB} \mathrm{~cm}^{-1}$ could be achieved with the implantation of realistic concentrations of $\sim 10^{19} \mathrm{~cm}^{-3}$ erbium. We have demonstrated that dislocation engineering can be used to significantly reduce the quenching of the optical gain with temperature enabling room temperature operation. These results suggest that we now have a realistic chance of achieving lasers and optical amplifiers in silicon itself.

Acknowledgements

This work was supported in part by the European Research Council under Grant 226470. 
Template_Ms Word preparing for Thin Solid Films, Special Issue of APAC-SILICIDE 2010

See details, Instructions to Authors, Elsevier web site

http://www.elsevier.com/wps/find/journaldescription.cws_home/504106/authorinstructions

\section{References}

[1] G.. Moore, Electronics 38 (1965) 1.

[2] M. A. Green, J. H. Zhao, A. H. Wang, P. J. Reece, M. Gal, Nature 412 (2001) 805.

[3] W. L. Ng, M. A. Lourenço, R. M. Gwilliam, S. Ledain, G. Shao, K. P. Homewood, Nature $410(2001) 192$.

[4] A. S. Liu, R. Jones, L. Liao, D. Samara-Rubio, D. Rubin, O. Cohen, R. Nicolaescu, M. Paniccia, Nature 427 (2004) 615.

[5] V. R. Almeida, C. A. Barrios, R. R. Panepucci, M. Lipson, Nature 431 (2004) 1081.

[6] O. Boyraz, B. Jalali, Opt. Express 12 (2004) 5269.

[7] H. S. Rong, A. S. Liu, R. Jones, O. Cohen, D. Hak, R. Nicolaescu, A. Fang, M. Paniccia, Nature 433 (2005) 292.

[8] H. S. Rong, R. Jones, A. S. Liu, O. Cohen, D. Hak, A. Fang, M. Paniccia, Nature 433 (2005) 725.

[9] S. G. Cloutier, P. A. Kossyrev, J. Xu, Nature Materials 4 (2005) 887.

[10] M. A. Foster, A. C. Turner, J. E. Sharping, B. S. Schmidt, M. Lipson, A. L. Gaeta, Nature 441 (2006) 960.

[11] R. S. Jacobsen, K. N. Andersen, P. I. Borel, J. Fage-Pedersen, L. H. Frandsen, O. Hansen, M. Kristensen, A. V. Lavrinenko, G. Moulin, H. Ou, C. Peucheret, B. Zsigri, A. Bjarklev, Nature 441 
Template_Ms Word preparing for Thin Solid Films, Special Issue of APAC-SILICIDE 2010

See details, Instructions to Authors, Elsevier web site

http://www.elsevier.com/wps/find/journaldescription.cws_home/504106/authorinstructions

(2006) 199.

[12] A. W. Fang, H. Park, O. Cohen, R. Jones, M. J. Paniccia, J. E. Bowers, Opt. Express 14 (2006) 9203.

[13] L. T. Canham, Appl. Phys. Lett. 57 (1990) 1046.

[14] L. T. Canham, T. I. Cox, A. Loni, A. J. Simons, Appl. Surface Sci. 102 (1996) 436.

[15] R. Zachai, K. Eberl, G.Abstreiter, E. Kasper, H. Kibbel, Phys. Rev. Lett. 64 (1990) 1055.

[16] L. Pavesi, Materials Today 1 (2005) 18.

[17] P. M. Fauchet, Materials Today 1 (2005) 26.

[18] J. Michel, J. L. Benton, R. F. Ferrante, D. C. Jacobson, D. J. Eaglesham, E. A. Fitzgerald,

Y. H. Xie, J. M. Poate, L. C. Kimerling, J. Appl. Phys. 70 (1991) 2672.

[19] S. Lombardo, S. U. Campisano, G. N. Vandenhoven, A. Cacciato, A. Polman, Appl. Phys.

Lett. 63 (1993) 1942.

[20] A. Polman, G. N. Vandenhoven, J. S. Custer, J. H. Shin, R. Serna, P. F. A. Alkemade, J.

Appl. Phys. 77 (1995) 1256.

[21] J. H. Shin, G. N. Vandenhoven, A. Polman, Appl. Phys. Lett. 67 (1995) 377.

[22] D. Leong, M. Harry, K. J. Reeson, K. P. Homewood, Nature 38 (1997) 686.

[23] C. M. Sun, H. K. Tsang, S. P. Wong, W. Y. Cheung, N. Ke, M. A. Lourenço, K. P.

Homewood, Nucl. Instr. and Meth. B 267 (2009) 1081, and references therein. 
Template_Ms Word preparing for Thin Solid Films, Special Issue of APAC-SILICIDE 2010

See details, Instructions to Authors, Elsevier web site

http://www.elsevier.com/wps/find/journaldescription.cws_home/504106/authorinstructions

[24] J. I. Pankove, Optical Processes in Semiconductors, Dover Publications, New York, 1971,

p. 215.

[25] T. Trupke, M. A. Green, P. Wurfel, J. Appl. Phys. 93 (2003) 9058.

[26] M. A. Lourenço, M. Milosavljević, S. Galata, M. S. A. Siddiqui, G. Shao, R. M. Gwilliam,

K. P. Homewood, Vacuum 78 (2005) 551.

[27] E. Rotem, J. M. Shainline, J. M. Xu, Appl. Phys. Lett. 91 (2007) 051127.

[28] Y. Ishikawa, K. Wada, D. D. Cannon, J. F. Liu, H. C. Luan, L. C. Kimerling, Appl. Phys.

Lett. 82 (2003) 2044.

[29] J. Liu, X. Sun, D. Pan, X. Wang, L. C. Kimerling, T. L. Koch, J. Michel, Opt. Express 15

(2007) 11272.

[30] M. A. Lourenço, M. S. A. Siddiqui, R. M. Gwilliam, G. Shao, K. P. Homewood, Physica E

16 (2003) 376.

[31] M. Milosavljević, G. Shao, M. A. Lourenço, R. M. Gwilliam, K. P. Homewood, J. Appl.

Phys. 97 (2005) 073512.

[32] J. P. Hirth, J. Lothe, Theory of Dislocations, John Wiley \& Sons, New York, 1982, p. 63.

[33] B. Welber, C. K. Kim, M. Cardona, S. Rodriguez, Solid State Commun. 17 (1975) 1021.

[34] M. Milosavljević, M. A. Lourenço, M. S. A. Siddiqui, G. Shao, R. M. Gwilliam, K. P.

Homewood, Inst. Phys. Conf. Ser. 179 (2003) 99. 
Template_Ms Word preparing for Thin Solid Films, Special Issue of APAC-SILICIDE 2010

See details, Instructions to Authors, Elsevier web site

http://www.elsevier.com/wps/find/journaldescription.cws_home/504106/authorinstructions

[35] M. Milosavljević, M. A. Lourenço, G. Shao, R. M. Gwilliam, K. P. Homewood, Appl. Phys.

B 83 (2006) 289.

[36] M. Milosavljević, M. A. Lourenço, G. Shao, R. M. Gwilliam, K. P. Homewood, Nucl. Instr. and Meth. B 266 (2008) 2470.

[37] M. A. Lourenço, M. Milosavljević, R. M. Gwilliam, K. P. Homewood, Appl. Phys. Lett. 87

(2005) 201105.

[38] M. A. Lourenço, R. M. Gwilliam, K. P. Homewood, Appl. Phys. Lett. 92 (2008) 161108.

[39] M. A. Lourenço, R. M. Gwilliam, K. P. Homewood, Appl. Phys. Lett. 91 (2007) 141122.

[40] N. Hamelin, P. G. Kik, J. F. Suyver, K. Kikoin, A. Polman, A. Schonecker, F. W. Saris, J.

Appl. Phys. 88 (2000) 5381.

[41] S. Saito, Jpn. J. Appl. Phys. 34 (2001) 236. 


\section{Figure captions}

Fig. 1. Comparison of photoluminescence emission from (a) undoped, (b) Tm doped and (c) Er doped samples with and without dislocation loops induced by B implantation. The paramount importance of the dislocation loops in enabling light emission is clear.

Fig. 2. Electroluminescence from a thulium doped DELED operating under forward bias at $80 \mathrm{~K}$.

Emission is observed over the 1.2 to $1.4 \mu \mathrm{m}$ wavelength region of the optical communications band.

Fig. 3. Photoluminescence (dotted line) due to the probe laser (pump laser off) from an Er-doped silicon waveguide and the change in the PL due to the probe (solid line), magnified by 100, induced by the pump laser (probe and the pump lasers on). Inset is an expansion of the 1500 to $1700 \mathrm{~nm}$ region showing the gain region due to the erbium.

Fig. 4. (a) Normalized change in the material gain $\Delta \mathrm{g}$, measured at $1550 \mathrm{~nm}$ as a function of temperature. The measurements have been made on two Er doped Si-on-Insulator (SOI) waveguide structures. One waveguide $(\boldsymbol{\Lambda})$ has been doped with erbium only in the silicon over layer and contains no dislocation loops; the second one ( $\square$ ) has been doped with erbium but also has a boron-induced dislocation loop array between the erbium and the silicon air interface. The importance of the loops in reducing the thermal quenching of the gain is clear. The residual quenching is attributed to the fact that some of the optically pumped erbium resides in the tail of the implant above the dislocation loops. The solid line is a guide for the eyes. (b) $\Delta \mathrm{g}$ measured at $80 \mathrm{~K}$ 
Template_ Ms Word preparing for Thin Solid Films, Special Issue of APAC-SILICIDE 2010

See details, Instructions to Authors, Elsevier web site

http://www.elsevier.com/wps/find/journaldescription.cws_home/504106/authorinstructions

as a function of power density for the same samples. The solid lines are theoretical fits to the data. 
Template_Ms Word preparing for Thin Solid Films, Special Issue of APAC-SILICIDE 2010

See details, Instructions to Authors, Elsevier web site

http://www.elsevier.com/wps/find/journaldescription.cws_home/504106/authorinstructions
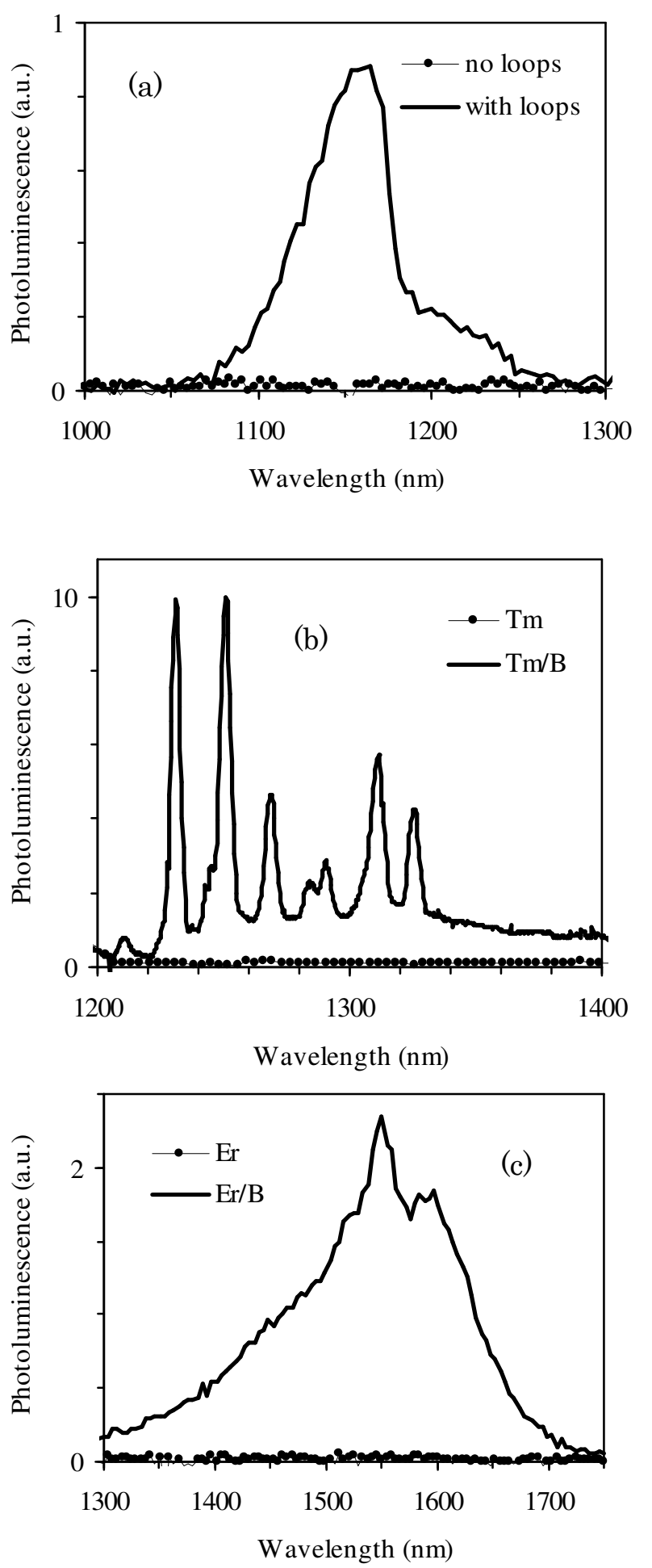

Fig. 1 
Template _ Ms Word preparing for Thin Solid Films, Special Issue of APAC-SILICIDE 2010

See details, Instructions to Authors, Elsevier web site

http://www.elsevier.com/wps/find/journaldescription.cws_home/504106/authorinstructions

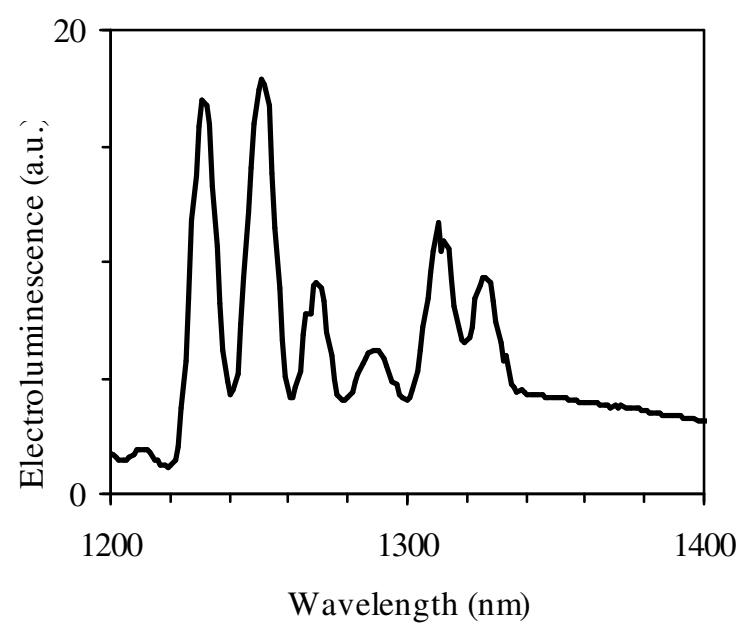

Fig. 2 
Template_Ms Word preparing for Thin Solid Films, Special Issue of APAC-SILICIDE 2010

See details, Instructions to Authors, Elsevier web site

http://www.elsevier.com/wps/find/journaldescription.cws_home/504106/authorinstructions

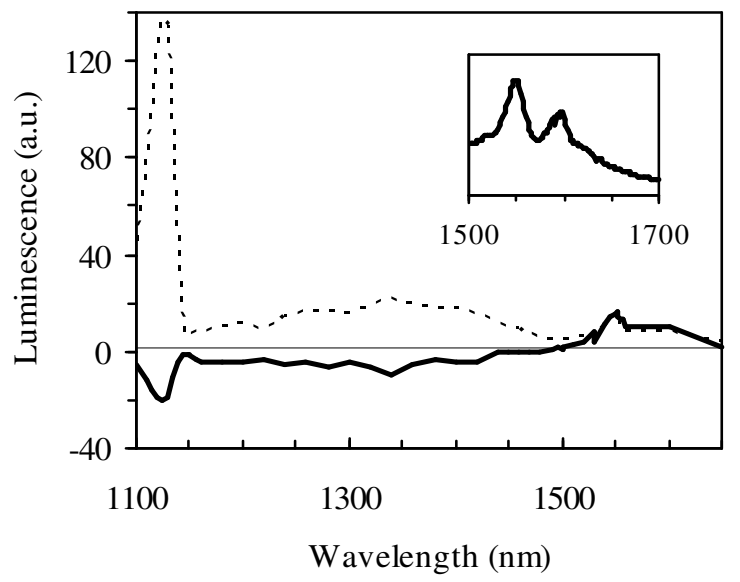

Fig. 3 
Template_Ms Word preparing for Thin Solid Films, Special Issue of APAC-SILICIDE 2010

See details, Instructions to Authors, Elsevier web site

http://www.elsevier.com/wps/find/journaldescription.cws_home/504106/authorinstructions
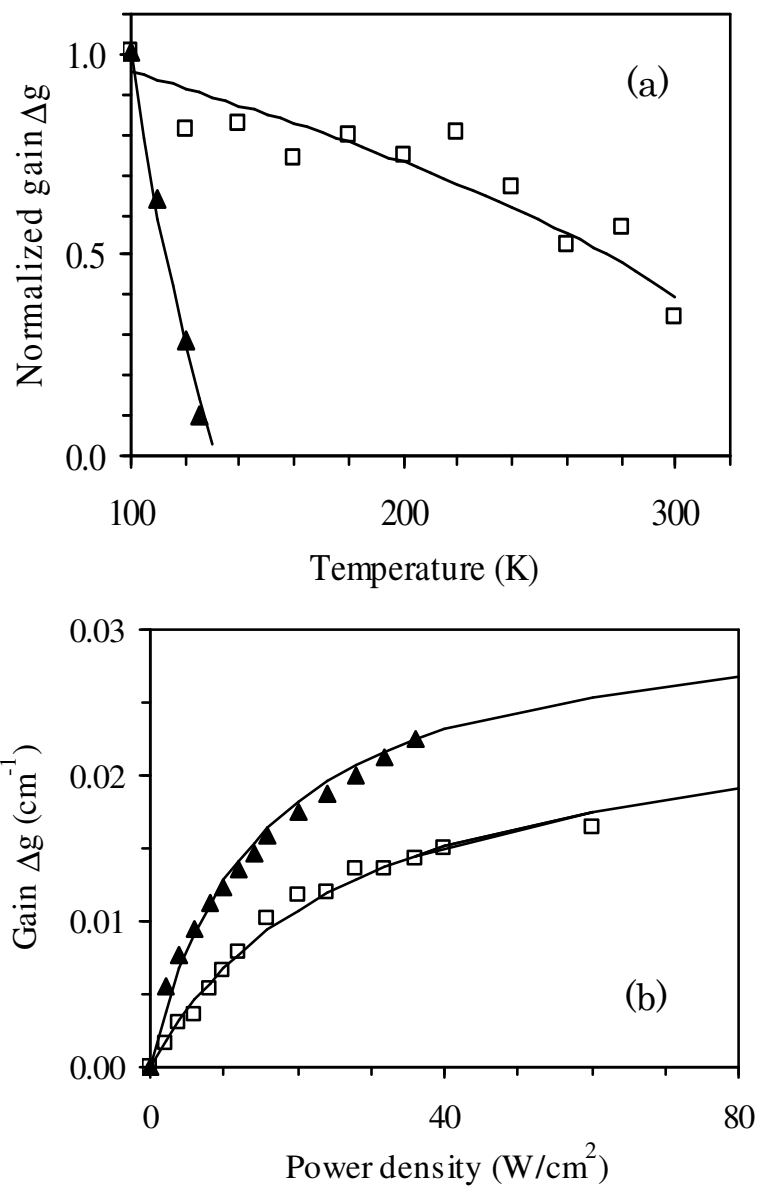

Fig. 4 